\title{
Antimicrobial Adjuvants Drug Discovery, the Challenge of Avoid the Resistance and Recover the Susceptibility of Multidrug-Resistant Strains
}

Juan Bueno*

Bioprospecting Development and Consulting, Bogotá, Colombia

\begin{abstract}
Currently, the emergence of multidrug resistant microorganisms as Acinetobacter baumannii and Mycobacterium tuberculosis as well as new drug resistant forms of Neisseria gonorrhoeae in conjunction with the evolving of resistance genes as the KPC (class A Klebsiella pneumoniae) and NDM-1 (New Delhi metallo-B-lactamase 1) carbapenemases are a public health threat that requires great efforts in prevention, treatment and diagnosis. For that reason, the InterAcademy Panel and InterAcademy Medical Panel statement (IAP-IAMP) included in their Global recommendations call for action to tackle antimicrobial resistance under the following item "Encourage industry innovation and public-private collaborative research and development programs for therapeutics, diagnostics, and vaccines". In this way, the development of novel antimicrobial drugs is not only necessary, is a priority the search and development of new pharmacological strategies for prevent antimicrobial resistance emergence, these strategies should have the ability of improve the current anti-infective therapy in combination, and recuperate the susceptibility of multidrug-resistant strains to the antibiotics. Thereupon, the introduction of screening platforms in a rational in vitro antimicrobial adjuvants drug discovery program is an important approach that will allow the detection of new chemical entities that can inhibit resistant mechanisms specifically and without side effects, looking for increase the microbicidal effect and prevent the development of subsequent mutations to anti-infective therapy. The aim of this review is to explore antibiotic adjuvants as therapeutic plan of action that can be developed for the discovery of new compounds that make bacteria more susceptible to anti-infectives by improving their efficacy.
\end{abstract}

Keywords: Antimicrobial resistance; Adjuvants; Drug discovery; Screening platforms

\section{Introduction}

Antibiotic-resistant strains are increasingly prevalent in hospitals and the community. Between antibiotic-resistant agents the major threats are constituted by methicillin-resistant Staphylococcus aureus (MRSA) that cause $\sim 19,000$ deaths by year in the U.S with a health care cost of \$3-4 billion. Equally, multidrug-resistant (MDR) and pan-drugresistant (PDR) gram-negative bacteria as Acinetobacter baumannii, Escherichia coli, Klebsiella pneumoniae, Pseudomonas aeruginosa, MDR and extensively-drug-resistant (XDR) strains of Mycobacterium tuberculosis (MDR-TB and XDR-TB) have increased cases in recent years persistently [1]. Therefore, the development of new antimicrobial agents is required to fight against these pathogens, but unfortunately the outcome in this regard has been slow, which suggests that strategies to discover new drugs should be reanalyzed [2]. Also, an important concern for to be considered is that acquired resistance is an adaptive response to the drug, because the indiscriminate use of antibiotics can cause selective pressure that induce resistance mechanism activation in microbes, which continue for many generations [3-5]. In this order of ideas antibiotics cause adaptive responses in microorganisms known as SOS repair system which not only acting on DNA also on the cell wall $[4,6]$.

The most known mechanisms of resistance in microbes are increase of drug efflux pumps, enzymatic inactivation, target site modification, and biofilm formation $[7,8]$, in response to environmental aggressions as well as anti-infective therapies $[9,10]$. For to decrease the emergence and transmission of antibiotic resistance, it is imperative to develop new anti-infective drugs with the ability of to inhibit drug efflux pumps as well as quorum sensing process and biofilm, also know more about biological mechanisms of induced resistance [11,12]. Equally, in the surveillance and prevention of the appearance of nonheritable resistance, is important to deep in the ways of antibiotic tolerance [13-15]. In the same way, employ in vitro pharmacokinetic/ pharmacodynamic models to improve and control dosing regimens may decrease the emergence of resistance during anti-infective therapy [16-18]. Reduce the consequences of antibiotic resistance requires a multidisciplinary view in a translational medicine program, that includes surveillance of rational use of antimicrobial agents and control of the dissemination of resistant microorganisms between hospitals and the community, likewise develop strategies that involving approaches from the pharmacokinetics, pharmacodynamics and pharmacogenomics $[19,20]$. For that reason an interesting approach would be discover new chemical entities that inhibits antimicrobial resistance mechanisms, consigned in a rational program of antimicrobial adjuvants drug discovery with the aim of develop new drug combinations that permits new antibiotic strategies in clinical practice $[21,22]$.

\section{Antibiotic adjuvants}

Among the therapeutic strategies that can be developed, antibiotic adjuvants, considered as compounds that make bacteria more susceptible to anti-infective by improving their efficacy is an interesting approach [23]. Adjuvants can be antibiotics, non-antibiotics, and molecules that can inhibit resistance genes and adaptive responses in microbial cells [24]. This synergism with antimicrobial activity can happen in several

*Corresponding author: Juan Bueno, Bioprospecting development and consulting Bogotá, Colombia, Tel: 571-3108323975; E-mail: juangbueno@gmail.com

Received February 16, 2016; Accepted March 01, 2016; Published March 08 2016

Citation: Bueno J (2016) Antimicrobial Adjuvants Drug Discovery, the Challenge of Avoid the Resistance and Recover the Susceptibility of Multidrug-Resistant Strains. J Microb Biochem Technol 8: 169-176. doi: 10.4172/1948-5948.1000281

Copyright: @ 2016 Bueno J. This is an open-access article distributed under the terms of the Creative Commons Attribution License, which permits unrestricted use, distribution, and reproduction in any medium, provided the original author and source are credited. 
ways such as: inhibition of antibiotic resistance elements involved in SOS response (DNA repair, mutagenesis) [25], increase in income of the antibiotic into the cell through the membrane, efflux pumps inhibition and altering the biological adaptive capacity of resistant cells [26]. For to do this, antibiotics can be combined with other molecules that are not, but having the ability to inhibit these resistance mechanisms as are: $\beta$-lactamases, cell wall thickening, biofilm formation, and efflux protein activation (Figure 1) [20]. Therefore, the design and development of antibiotic adjuvants can increase the microbicidal effect and prevent the development of subsequent mutations to anti-infective therapy [26]. This combination strategy have been demonstrated as in the case of trimethoprim and sulfa drugs [27], and introduction of $\beta$-lactamase inhibitors (clavulanate, sulbactam and tazobactam) [28]; more recently a mixture of cefuroxime and ticlopidine with synergistic activity against MRSA have been demonstrated, increasing the antimicrobial activity of cephalosporins [29].

\section{Antimicrobial synergism}

In this order of ideas for fight against infections caused by MDR microorganisms is necessary develop new therapies that consider the combination of various medications during the treatment time, taking into account the potential drug interactions during the drug development process. This strategy has been used successfully in anticancer treatment, HIV infections, complicated cases of malaria and the treatment of Mycobacterium tuberculosis infections [20], in clinical medicine this approach is routinely employed in order to obtain clinical improvement in difficult to cure infections. Nevertheless despite this, side effects are present, for that reason the combination between antibacterials and nontoxic antimicrobial adjuvants can be an interesting choice in search of a new type of synergism combination therapy whose main objective is to provide anti-infective medical care without causing resistance. The principal targets of these adjuvants for develop synergistic interactions would be microbial signaling, microbial

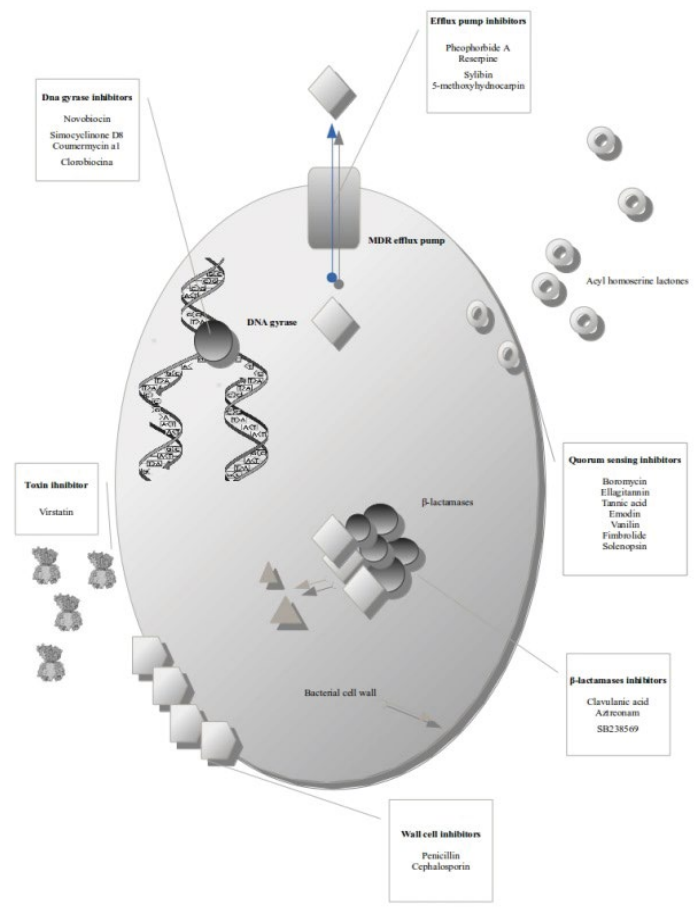

Figure 1: Antimicrobial targets for adjuvants and inhibitors. effector molecules, virulence factors, resistance gene expression and horizontal transfer [30].

\section{Antimicrobial targets for antimicrobial adjuvants}

Using current methodologies of molecular biology has been possible to identify $265-350$ anti-bacterial targets. Of which $60 \%$ are present in every pathogenic bacteria, but no more than 20 can be inhibited by the drugs available on the market [31]. For that reason, antimicrobial drug screening have been focused in antimicrobial target discovery, leaving aside whole cell assays that can identify molecules that can cross cellular barriers and inhibit biological processes [32]. In consequence, in vitro cell free screening was not successful [31]. So it is now recommended, combine antimicrobial targets approach with whole cell assays, with the end of increase the detection of compounds able to inhibit microbial cultures with a mechanism of action determined [33]. In this way, in the following excerpts the intention is to describe what would be the appropriate targets to develop a platform of this type, together with the corresponding phenotype cell assay.

\section{Antibiotics combination FIC index}

For determinate the susceptibility of bacteria and fungi to new antimicrobial products is necessary apply a uniform terminology based in the standards set forth in European Committee for Antimicrobial Susceptibility Testing (EUCAST) of the European Society of Clinical Microbiology and Infectious Diseases (ESCMID) and Clinical and Laboratory Standard Institute (CLSI) from USA. Using these standards is possible determinate four antimicrobial activities of the products in combination: indifference, additive, synergism and antagonism. The observed increase in activity always has to be confirmed using timekill curve methods for to analyze the synergism and their bactericidal activity. Finally, the fractional inhibitory concentration (FIC) index is an important predictor to begin the evaluation of promising antimicrobial adjuvants in combination [34].

\section{Cell Wall and Membrane}

\section{Enhancing the permeability of the cell wall}

The initial bacterial defense mechanism is the control of membrane permeability with the end of decrease the intracellular concentration of antibiotics and biocides [35]. Equally, in gram-negative bacteria has been demonstrated that the outer membrane permeation is an initial step in the bactericidal activity of antimicrobial compounds [36]. Constituting the membrane disruption as a key target in the development of new drugs with potent bactericidal without emergence of resistance [37]. The membrane disruption can be obtained using essential oils, detergents, surfactants, polymyxins, daptomycin and antimicrobial peptides $[38,39]$. This target can be promising for the development of new antiseptics and disinfectant agents for eradicate MDR microorganisms from hospital environment [40].

\section{Efflux pump inhibitors}

One of the biological systems that control the intracellular concentration of anti-infective drugs are efflux pumps, which makes the search for molecules with efflux pump inhibition (EPIs) activity an important strategy to restore antimicrobial activity of existing antibiotics. The families of efflux transporters are composed by MFS (major facilitator superfamily), MATE (multi-drug and toxic compound extrusion), RND (resistance nodulation cell division) superfamily, SMR (small multi-drug-resistance), and ABC (ATP-binding cassette) transporters [41]. EPIs can be used inhibiting several of these efflux transporters families as adjuvants in antibiotic treatments affecting 
bacterial survival, virulence and pathogenicity [42]. In this way, the efflux pump inhibitor verapamil has been evaluated using in vivo models as an adjuvant showing activity in antimicrobial treatment and increasing bactericidal activity $[43,44]$.

\section{ROS production induction}

It has been observed in several studies that bactericidal antibiotics cause cell destruction by production of reactive oxygen species (ROS) [45]. ROS can damage DNA, RNA, proteins and lipids. So it has been proposed as strategy to increase antibiotic efficacy induce ROS production in microbial cells as well as inhibit detoxification and repair systems [23,46]. Likewise, is an important approach take advantage of the oxidative stress response for improving anti-infective therapies, giving way to the development of a new class of molecules [47]. Interestingly, there is connection between oxidative stress response and anti-infective drug action regulated by SoxRS and give antimicrobial resistance, which explain why antioxidants, such as vitamin $\mathrm{C}$ and glutathione increase antimicrobial activity of quinolones and aminoglycosides and supress mutagenesis that produce quinolone resistance $[48,49]$.

\section{Virulence Factors Inhibition}

\section{Toxin inhibition}

The production of toxins is an important virulence factor in bacteria and fungi. That target host cells by binding to specific glycolipids in the cell membrane. The inhibition of this interaction can prevent the potential tissue damage [50]. The most common microbial toxins described are: aflatoxin B1, fumonisins, and ochratoxin A produced by fungi; cholera toxin produced by Vibrio cholerae bacteria; Shiga toxins produced by $E$. coli bacteria; staphylococcal enterotoxins produced by Staphylococcus aureus bacteria and listeriolysin O (LLO) produced by Listeria monocytogenes [51]. This anti-virulence strategy looking for protects hosts cells against the adverse effects of toxins is an innovative approach for the control of infection and decrease the development of resistance [52]. The disadvantage of use of toxin inhibitors can be the toxin over-expression, increasing the virulence [53]. In this way, the most promising compound detected has been virstatin that inhibits ToxT dimerization of cholera toxin in Vibrio cholerae [54].

\section{Anti-betalactamase}

$\beta$-lactamases are enzymes that catalyze the hydrolysis of $\beta$-lactam ring of the antibiotics inactivating. Up to date, more than 700 of these enzymes have been detected and are classified according to the type of target antibiotic in four groups, A-D (class A penicillins, class C cephalosporins, class D oxacillin and class B for have two zinc ions at its catalytic site are called metallo- $\beta$-lactamases) [55]. Thanks to the success obtained with clavulanic acid, it is now a well-established principle that the combination of a $\beta$-lactamase inhibitor (as an adjuvant) with a $\beta$-lactam antibiotic enhances the efficacy. For that reason, there is an interesting research field in the development and search of novel $\beta$-lactamase inhibitors for co-administration with $\beta$-lactams [56].

\section{Anti-quorum sensing}

Bacterial communication is developed across production and detection of signaling molecules which belongs to quorum sensing (QS) process that regulates the formation of virulence factors, antibiotic production and biofilm formation [57]. A compound with anti-QS activity should be able of inhibit signal biosynthesis, block activity and decrease production of acyl homoserine lactones (AHL), induce enzymatic signal degradation and inhibit the reception of signal molecules [58], as well as mimic AHL for induce altered response [59].
Therefore, QS is a fascinating target for the development of novel antiinfective medications in combination with antibiotics [57].

\section{Replication and Resistance Gene Transfer DNA synthesis}

DNA gyrase and topoisomerase IV are crucial in DNA synthesis, constituting in important therapeutic targets. Because, ATP-dependent supercoiling of DNA is catalyzed by DNA gyrase being essential in DNA replication, transcription, and recombination and in the regulation of chromosome supercoiling [60]. In this way, fluoroquinolones have the ability of inhibit bacterial DNA gyrase and topoisomerase IV binding to the enzyme-DNA complex in most bacterial species causing cell death by the accumulation of double-stranded DNA fragments. Currently the challenge is developing and discovers new chemical entities better than fluoroquinolones, but do not develop resistance [61].

\section{Gene transfer inhibition}

Horizontal gene transfer is defined as transportation of DNA fragments from one organism to another and subsequent incorporation, causing changes in their genetic structure. This DNA transfer is considered a critical mechanism whereby antibiotic resistance and virulence factors are acquired in bacterial communities. The pharmacological inhibition of transfer can be a crucial approach in the blocking of antimicrobial resistance spread, in this order of ideas antibiotics, polycyclic chemicals, and crude extracts have shown the ability of inhibit the transportation of the DNA fragments with resistance mechanisms in bacteria, preventing propagation genetic and making them more susceptible to the action of antibiotics [62].

\section{Antiplasmid}

The eradication of plasmids that transport antibiotic resistant genes can turn again microbial populations in susceptible to antimicrobials and recovering the spectrum of activity [63]. Drugs such as promethazine and peppermint oil and its main constituent, menthol have eliminated plasmids that carry resistance genes $[63,64]$, which makes compounds with antiplasmid activity as interesting adjuvant antimicrobial agents in the fight against drug resistance.

\section{Antimicrobial Adjuvants Drug Screening Program}

Is necessary the search and development of new pharmacological strategies for prevent antimicrobial resistance emergence, these strategies should have the ability of improve the current anti-infective therapy in combination, and recuperate the susceptibility of multidrug-resistant strains to the antibiotics. In this way, the introduction of screening platforms looking for a rational in vitro antimicrobial adjuvants drug discovery is an important approach that will allow the detection of new chemical entities that can inhibit resistant mechanisms specifically and without side effects [65]. In total should be implemented a mode of action (MOA) screening platform in combination with whole cell assays for natural and chemical library evaluation (Figure 2) [66].

\section{Antimicrobial screening platforms to detect drugs that target microbial cell wall or membrane}

The fast development of resistance makes many therapeutic targets lose their clinical efficacy. The envelope of Gram-negative species and mycobacteria presents a barrier for penetration, making it difficult to discover broad spectrum compounds. Increasing penetration of a large set of compounds and ranking them will enable the deduction of rules of penetration [67]. Thus, are necessary implement new agents that increase the permeability of the bacterial cell membrane, 


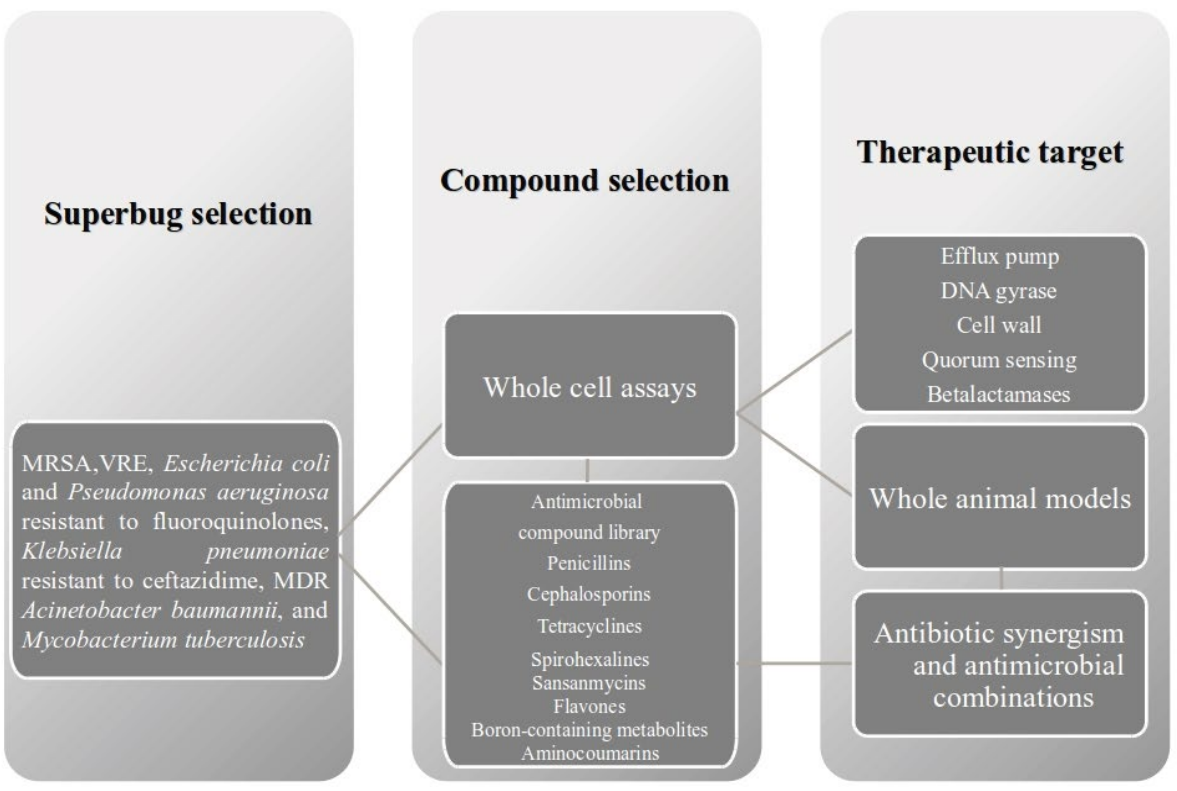

Figure 2: Integrative model in an antimicrobial adjuvant drug screening program.

which will make the majority of targets available to drug effects [37,67]. This strategy should be focused in three screening platforms looking for cell permeabilizers and efflux pump inhibitors and ROS production inducers, with the end of identify molecules that increase the penetration of antibiotics and keep the intracellular concentrations, decreasing antibiotic resistance emergence [68].

\section{Antimicrobial screening platforms to detect drugs that target microbial virulence factors}

Have been demonstrated that targeting key virulence factors can improve the healing in infectious models in vivo. The combination of an anti-virulence agent with a conventional antibiotic to achieve synergism is necessary for avoid the resistance selection [53]. Antivirulence drugs are an important research field that can impact in drug development enhancing the anti-infective drug efficacy. These anti-virulence drugs decrease the pathogenicity of the etiological agent weakening the selection for resistance induced by antibiotics [69] This strategy should be focused in three screening platforms looking for toxin inhibitors, biofilm and quorum sensing inhibitors and antibetalactamase compounds, with the end of identify new molecules with anti-virulence activity [70].

\section{Antimicrobial screening platforms to detect drugs that inhibit DNA replication and gene transfer}

Microorganisms use mutation and horizontal gene transfer for diversify its genome. Antibiotic treatment stimulates mutagenesis in microbial populations; these mutations confer antibiotic resistance and can be disseminated to other individuals. For that reason is important identify new chemical entities that block the selection of the resistant mutants and further growth [71]. The development, acquisition and spread of antibiotic resistance in clinical environments have high impact in the clinical outcome to antimicrobial therapy around the world. The resistance acquired genetically by horizontal gene transfer or gene mutation is manifested phenotypically by target modification and over-expression of efflux pumps, blocking the transfer of genetic information is possible to reuse antibiotic resistance that have become obsolete [72]. This strategy should be focused in antiplasmid screening platforms and DNA gyrase inhibition looking for obtains non-antibiotic adjuvants that become susceptible the MDR microorganisms to low concentrations of antibiotics. Equally, mutant prevention concentration (MPC) testing that represents the minimal dose that prevents the emergence of resistant mutants and mutant selection window of the compounds in combination should be performed [73].

Antimicrobial screening platforms to detect drugs that inhibit bacterial dormancy and persistence

Microbial persisters are tolerant to several bactericidal antibiotics; this phenomenon is named multidrug tolerance (MDT). These persisters not killed by antibiotics could revert to replicating forms (reverters), producing recurrent infections, for that reason is necessary new antimicrobial drugs with bactericidal activity against persisters cells [74]. Persisters are bacterial cells that are multidrug tolerant as a consequence of a transient switch [75]. Persister cells are responsible for the intractability of many bacterial infections to antibiotic treatment, which often result in recurrent courses of antibiotics [76]. Also, dormancy in biofilms and persister cells presence are the cause of the reduced susceptibility of biofilms to antibiotics [77]. This strategy should be focused in develop type I and type II persister cell assays of exponential- and stationary-phase cultures, for detection of antipersisters chemical entities [78]. Equally, must be implemented an in vitro model of mycobacterial dormancy with colorimetric methods for determinate anti-dormancy and anti-tubercular activity [79].

In vitro whole animal infection model using $C$. elegans antimicrobial screening platforms to evaluate antimicrobial activity and determine toxicity

Drug discovery is a challenging research field. Research with the use of Caenorhabditis elegans has demonstrated great results and holds a promising future. The nematode is an excellent tool for the researcher and provides many valuable experimental elements. The 
many advantages of their use, including easy handling, the option for high throughput assays and the possibility of study of the interactions between the host and the pathogen have made the C. elegans a promising tool for preclinical drug discovery [80]. The nematode $C$. elegans is a whole animal model system for identifying small molecules with in vivo anti-infective properties. C. elegans can be infected with a broad range of human pathogens (Enterococcus faecalis, Staphylococcus aureus, Pseudomona aeuroginosa, Salmonella enterica and Candida albicans) $[81,82]$. Because of their small size, C. elegans nematodes can be placed in 96- or 384-well plates for high throughput experiments. This model offers distinct advantages for antimicrobial discovery as it provides the possibility to identify compounds with no antimicrobial activity in vitro, but which may target functions only important for in vivo survival or virulence [83].

Evaluate antimicrobial activity against MDR microorganisms using the screening platforms implemented of non-antibiotics compounds, essential oils as well as their formulations and derivate in combination with existing antibiotics

Non-antibiotics and essential oils have demonstrated to enhance the activity of antimicrobial drugs [83,84], also, phenothiazines and essential oils possess plasmid inhibition activities which can stop the transmission of resistance to other populations [64,85], examining the response of MDR microorganisms against these combinations is possible induce that we can be in the presence of a therapeutic alternative for treatment of these diseases which are destroying thousands of lives per year throughout the world. In previous reports it has been suggested that phenothiazines can be used as antimicrobial adjuvants. Between them chlorpromazine and amitriptyline have reduced or reversed resistance of clinical isolates to penicillin, tobramycin and cefuroxim [86]. Also, promazine, promethazine, prochlorperazine, methdilazine, fluphenazine, trimeprazine, trifluoperazine, triflupromazine, thioridazine and flupenthixol have shown antimicrobial activity against a broad spectrum of microorganisms $[85,87]$. Equally there are reports about the activity of essential oils as antibiotic resistance-modifying [88]. Essential oils from Pelargonium graveolens, Lantana camara, Hyptis martiusii and Croton zehntneri have shown enhance the antimicrobial activity against resistant microorganisms in combination with other molecules as aminoglycosides (amikacin, kanamycin and gentamicin) so they have potential to be used as antimicrobial adjuvants $[89,90]$. This strategy should be focused in the evaluation of promising antimicrobial adjuvants in combination looking for clinical application, for this aim will be used the previously implemented screening platforms and will employ non-antibiotics drugs, essential oils both new formulations and their derivatives.

\section{The Importance of Natural Products as Source of New Compounds Chemically Diverse in Antimicrobial Adjuvants Discovery}

So et al. [91] described the bottlenecks in R\&D pipeline for novel antibacterial drugs and categorized in the following items:

\section{Lead identification}

Currently is considered that high throughput screening approach for novel antimicrobial drug candidates has had very little performance in comparison to other public health problems. Because, the current whole cell drug screens have been unsuccessful [82].

\section{Medicinal chemistry}

Since the proposal of rational anti-infective chemotherapy through the search for selective inhibitors of microbial enzymes, the process of description and development in to clone genes and manipulate bacterial strains have achieved to enhance whole-cell phenotype screens for inhibitors of specific targets, and become antimicrobial drug discovery toward screening target-directed, seeking to increase the druggability [92]. But, slowing the process of transforming these leads into drugs that can enter in controlled clinical trials.

\section{Crossing the valley of death}

In translational research this term is used for describe the chasm caused by the absence of communication between clinical and basic scientists that prevents those discoveries of biomedical researchers reach to the patients [93]. In this valley the successful depends of control of risks: Scientific, Intellectual Property, Market, and Regulatory [94]. In antimicrobial drug discovery the success during lead optimization depends largely in the medicinal chemistry approach developed, and this influences the costs $[93,94]$.

\section{Regulatory approval}

Types of clinical trials as well as enrolling adequate numbers of patients should be identified for to control costs and guidelines of regulatory agencies should be discussed [95].

\section{Reimbursement}

Reimbursement is a controversial aspect of antibiotic development especially for the duration of treatment (10-14 days), for that reason is very important look for a balance between research, regulation and economics [14,91].

Natural products for antibacterial drug screening has had a significant decrease in the obtainment of new leads, since the advent of high throughput screening methods, and the increase in its replacement of screening of chemical libraries from combinatorial chemistry [96]. Limiting antibacterial discovery to screening of this material, although the chemical diversity of natural products is greater [97-99]. Where soil has been the most explored environment with the highest number of compounds isolated in the search for natural products with antimicrobial activity [100]. Within this diversity the largest producers of antimicrobials are streptomyces, bacillus, and myxococcus species [98]. Microorganisms have been the largest natural source of drugs used at present, in where $45 \%$ are produced by actinomycetes, $38 \%$ by fungi and $17 \%$ by unicellular bacteria $[101,102]$. Berdy [103] has described that the currently decrease in the research in isolation of new microbial metabolites could be due to the mix of the following factors:

- Human factor (disparagement of the microbes by the use of chemical libraries).

- Scientific mistake (due to constraints of high throughput screening methods and the absence of chemical diversity of the compounds from combinatorial synthesis).

- Costs and regulations of an antimicrobial drug discovery program [103].

To solve the weaknesses of search of bioactive microbial metabolite and optimize the rate of the discoveries is important to develop the following:

Seek new promising sources of natural products (e.g. Endophytes, essential oils) [104,105].

Develop new screening methods more selective and effective, with the ability of predict clinical response of the selected compounds [106]

Implement and use other models for study pathogenesis and therapeutics of infectious diseases [107]. 
Citation: Bueno J (2016) Antimicrobial Adjuvants Drug Discovery, the Challenge of Avoid the Resistance and Recover the Susceptibility of MultidrugResistant Strains. J Microb Biochem Technol 8: 169-176. doi: 10.4172/1948-5948.1000281

Equally, is necessary to develop new drugs that acting not necessarily to eliminate the infectious agent in the individual patient, also for to prevent antibiotic resistance in specific environments weakening the evolutionary possibilities of the biological elements involved as it is chemical communication, which makes different microorganisms deliver a coordinated response to environmental stimuli [108-110].

\section{Conclusion}

Antimicrobial drug resistance is one of the greatest threats to the health of people, and antibiotic discovery is a growing need. In this way is necessary design strategies in screening for the detection and development of anti-infective, but these new approaches require combination of target-based activity with whole cell inhibition, with the end of obtain antimicrobial drugs with novel mechanisms of action. Antibiotics adjuvants that use the mechanisms involved in antibiotic resistance or virulence is a promising approach for new "magic bullets" and prevents the emergence of resistance. Also, this strategy is complementary to the pursuit of synergistic combinations. Currently, the principal limitation of studies in drug is the selection of bacterial strains and determinate the induction of mutation developed by drugs. In this research field the design of the antimicrobial screening strategy is focused in the detection of drugs that target microbial cell wall or membrane, microbial virulence factors, DNA replication and gene transfer, C. elegans infection model and bacterial dormancy and persistence, this in combination of clinical microbiology assays of antimicrobial susceptibility testing, checkerboard analyses, and mutant prevention concentration (MPC) for obtain a mutant selection window of the compounds in combination against MDR pathogens. Because the urgent need of new antibiotics require projects focused in application and development of new products that have shown activity as antimicrobial adjuvants with promising application in prevention of hospital-acquired infections. The challenges to be applied in this research will provide new therapeutic alternatives in the control and treatment in infectious diseases caused by MDR microorganisms. The concept of antibiotic adjuvants is an important strategy that look for recover the lost susceptibility of the etiological agents to the current anti-bacterial therapy, in this order of ideas the obsolete compounds can be used again in new formulations and combinations, because is necessary to remember that resistance is not an isolated phenomenon, it is a public health problem in all its aspects has the same causes that this exploration seeks to overcome into new products.

\section{References}

1. Fischbach MA, Walsh CT (2009) Antibiotics for emerging pathogens. Science 325: 1089-1093

2. Payne DJ, Gwynn MN, Holmes DJ, Pompliano DL (2007) Drugs for bad bugs: confronting the challenges of antibacterial discovery. Nat Rev Drug Discov 6: $29-40$

3. Li XZ, Nikaido H (2009) Efflux-mediated drug resistance in bacteria: an update. Drugs 69: 1555-1623.

4. Baquero F, Cantón R (2009) Evolutionary biology of drug resistance: Antimicrobial drug resistance. Springer-Verlag, Heidelberg.

5. Chen Z, Jiang X (2014) Microbiological safety of chicken litter or chicken litterbased organic fertilizers: a review. Agriculture 4: 1-29.

6. Andersson DI, Hughes D (2014) Microbiological effects of sublethal levels of antibiotics. Nat Rev Microbiol 12: 465-478.

7. Romero D, Traxler MF, López D, Kolter R (2011) Antibiotics as signal molecules. Chem Rev 111: 5492-5505.

8. Beceiro A, Tomás M, Bou G (2013) Antimicrobial resistance and virulence: a successful or deleterious association in the bacterial world? Clin Microbiol Rev 26: $185-230$
9. Allen HK, Donato J, Wang HH, Cloud-Hansen KA, Davies J, et al. (2010) Call of the wild: antibiotic resistance genes in natural environments. Nat Rev Microbiol 8: 251-259.

10. Nguyen D, Joshi-Datar A, Lepine F, Bauerle E, Olakanmi O, et al. (2011) Active starvation responses mediate antibiotic tolerance in biofilms and nutrientlimited bacteria. Science 334: 982-986.

11. Tommasi R, Brown DG, Walkup GK, Manchester JI, Miller AA (2015) ESKAPEing the labyrinth of antibacterial discovery. Nat Rev Drug Discov 14 529-542.

12. Cars O, Hedin A, Heddini A (2011) The global need for effective antibioticsmoving towards concerted action. Drug Resist Updat 14: 68-69.

13. Nathan C (2012) Fresh approaches to anti-infective therapies. Sci Transl Med 4: $140 \mathrm{sr} 2$.

14. Shlaes D, Projan S (2009) Antimicrobial resistance versus the discovery and development of new antimicrobials: Antimicrobial drug resistance. SpringerVerlag, Heidelberg.

15. Cohen NR, Lobritz MA, Collins JJ (2013) Microbial persistence and the road to drug resistance. Cell Host Microbe 13: 632-642.

16. Vaddady PK, Lee RE, Meibohm B (2010) In vitro pharmacokinetic/ pharmacodynamic models in anti-infective drug development: focus on TB. Future Med Chem 2: 1355-1369.

17. Mouton JW, Ambrose PG, Canton R, Drusano GL, Harbarth S, et al. (2011) Conserving antibiotics for the future: new ways to use old and new drugs from a pharmacokinetic and pharmacodynamic perspective. Drug Resist Updat 14: 107-117.

18. Trivedi A, Lee RE, Meibohm B (2013) Applications of pharmacometrics in the clinical development and pharmacotherapy of anti-infectives. Expert Rev Clin Pharmacol 6: 159-170.

19. Freire-Moran L, Aronsson B, Manz C, Gyssens IC, So AD, et al. (2011) Critical shortage of new antibiotics in development against multidrug-resistant bacteriaTime to react is now. Drug Resist Updat 14: 118-124.

20. Worthington RJ, Melander C (2013) Combination approaches to combat multidrug-resistant bacteria. Trends Biotechnol 31: 177-184.

21. Kalan L, Wright GD (2011) Antibiotic adjuvants: multicomponent anti-infective strategies. Expert Rev Mol Med 13: e5.

22. Fears R, ter Meulen V (2014) What do we need to do to tackle antimicrobia resistance? Lancet Glob Health 2: e11-12.

23. Farha MA, Brown ED (2013) Discovery of antibiotic adjuvants. Nat Biotechnol 31: $120-122$.

24. Allen HK, Trachsel J, Looft T, Casey TA (2014) Finding alternatives to antibiotics. Ann N Y Acad Sci 1323: 91-100.

25. Norton MD, Spilkia AJ, Godoy VG (2013) Antibiotic resistance acquired through a DNA damage-inducible response in Acinetobacter baumannii. J Bacterio 195: $1335-1345$

26. Bernal P, Molina-Santiago C, Daddaoua A, Llamas MA (2013) Antibiotic adjuvants: identification and clinical use. Microb Biotechnol 6: 445-449.

27. Chevereau G, Bollenbach T (2015) Systematic discovery of drug interaction mechanisms. Mol Syst Biol 11: 807

28. Drawz SM, Bonomo RA (2010) Three decades of beta-lactamase inhibitors Clin Microbiol Rev 23: 160-201.

29. Farha MA, Leung A, Sewell EW, D'Elia MA, Allison SE, et al. (2013) Inhibition of WTA synthesis blocks the cooperative action of PBPs and sensitizes MRSA to Î-lactams. ACS Chem Biol 8: 226-233.

30. Pieren M, Tigges M (2012) Adjuvant strategies for potentiation of antibiotics to overcome antimicrobial resistance. Curr Opin Pharmacol 12: 551-555.

31. Singh SB (2014) Confronting the challenges of discovery of novel antibacteria agents. Bioorg Med Chem Lett 24: 3683-3689.

32. Genilloud O (2012) Current challenges in the discovery of novel antibacterials from microbial natural products. Recent Pat Antiinfect Drug Discov 7: 189-204.

33. Genilloud O (2014) The re-emerging role of microbial natural products in antibiotic discovery. Antonie Van Leeuwenhoek 106: 173-188. 
Citation: Bueno J (2016) Antimicrobial Adjuvants Drug Discovery, the Challenge of Avoid the Resistance and Recover the Susceptibility of MultidrugResistant Strains. J Microb Biochem Technol 8: 169-176. doi: 10.4172/1948-5948.1000281

34. Abreu AC, McBain AJ, Simões M (2012) Plants as sources of new antimicrobials and resistance-modifying agents. Nat Prod Rep 29: 1007-1021.

35. Martínez de Tejada G, Sánchez-Gómez S, Rázquin-Olazaran I, Kowalski I, Kaconis $\mathrm{Y}$, et al (2012) Bacterial cell wall compounds as promising targets of antimicrobial agents I. Antimicrobial peptides and lipopolyamines. Curr Drug Targets 13: 1121-1130

36. Miki T, Hardt WD (2013) Outer membrane permeabilization is an essential step in the killing of gram-negative bacteria by the lectin RegIIII ${ }^{2}$. PLoS One 8: e69901.

37. Hurdle JG, O'Neill AJ, Chopra I, Lee RE (2011) Targeting bacterial membrane function: an underexploited mechanism for treating persistent infections. Nat Rev Microbiol 9: 62-75.

38. Patrone V, Campana R, Vittoria E, Baffone W (2010) In vitro synergistic activities of essential oils and surfactants in combination with cosmetic preservatives against Pseudomonas aeruginosa and Staphylococcus aureus. Curr Microbio 60: $237-241$.

39. Bolla JM, Alibert-Franco S, Handzlik J, Chevalier J, Mahamoud A, et al. (2011) Strategies for bypassing the membrane barrier in multidrug resistant Gramnegative bacteria. FEBS Lett 585: 1682-1690.

40. Laverty G, Gorman SP, Gilmore BF (2011) The potential of antimicrobial peptides as biocides. Int J Mol Sci 12: 6566-6596.

41. Mandal SM, Roy A, Ghosh AK, Hazra TK, Basak A, Franco OL (2014) Challenges and future prospects of antibiotic therapy: from peptides to phages utilization. Front Pharmacol 5: 105.

42. Bhardwaj AK, Mohanty $P$ (2012) Bacterial efflux pumps involved in multidrug resistance and their inhibitors: rejuvinating the antimicrobial chemotherapy. Recent Pat Antiinfect Drug Discov 7: 73-89.

43. Gupta S, Tyagi S, Bishai WR (2015) Verapamil increases the bactericidal activity of bedaquiline against Mycobacterium tuberculosis in a mouse model. Antimicrob Agents Chemother 59: 673-676.

44. Pule CM, Sampson SL, Warren RM, Black PA, van Helden PD, et al. (2016) Efflux pump inhibitors: targeting mycobacterial efflux systems to enhance TB therapy. J Antimicrob Chemother 71: 17-26.

45. Kohanski MA, Dwyer DJ, Collins JJ (2010) How antibiotics kill bacteria: from targets to networks. Nat Rev Microbiol 8: 423-435.

46. Brynildsen MP, Winkler JA, Spina CS, MacDonald IC, Collins JJ (2013) Potentiating antibacterial activity by predictably enhancing endogenous microbial ROS production. Nat Biotechnol 31: 160-165.

47. Dwyer DJ, Kohanski MA, Collins JJ (2009) Role of reactive oxygen species in antibiotic action and resistance. Curr Opin Microbiol 12: 482-489.

48. Zhao X, Drlica K (2014) Reactive oxygen species and the bacterial response to lethal stress. Curr Opin Microbiol 21: 1-6.

49. Zhao X, Hong Y, Drlica K (2015) Moving forward with reactive oxygen species involvement in antimicrobial lethality. J Antimicrob Chemother 70: 639-642.

50. Branson TR, Turnbull WB (2013) Bacterial toxin inhibitors based on multivalen scaffolds. Chem Soc Rev 42: 4613-4622.

51. Friedman M, Rasooly R (2013) Review of the inhibition of biological activities of food-related selected toxins by natural compounds. Toxins (Basel) 5: 743-775.

52. Sousa S, Mesquita FS, Cabanes D (2015) Old war, new battle, new fighters! J Infect Dis 211: 1361-1363.

53. Allen RC, Popat R, Diggle SP, Brown SP (2014) Targeting virulence: can we make evolution-proof drugs? Nat Rev Microbiol 12: 300-308.

54. Anthouard R, DiRita VJ (2013) Small-molecule inhibitors of toxT expression in Vibrio cholerae. MBio 4

55. Schlesinger SR, Lahousse MJ, Foster TO, Kim S-K (2011) Metallo-ßLactamases and Aptamer-Based Inhibition. Pharmaceuticals 4: 419-428.

56. Gill EE, Franco OL, Hancock RE (2015) Antibiotic adjuvants: diverse strategies for controlling drug-resistant pathogens. Chem Biol Drug Des 85: 56-78.

57. Koh CL, Sam CK, Yin WF, Tan LY, Krishnan T, et al. (2013) Plant-derived natural products as sources of anti-quorum sensing compounds. Sensors (Basel) 13: 6217-6228.
58. Khan MS, Zahin M, Hasan S, Husain FM, Ahmad I (2009) Inhibition of quorum sensing regulated bacterial functions by plant essential oils with special reference to clove oil. Lett Appl Microbiol 49: 354-360.

59. Corral-Lugo A, Daddaoua A, Ortega A, Espinosa-Urgel M, Krell T (2016) Rosmarinic acid is a homoserine lactone mimic produced by plants that activates a bacterial quorum-sensing regulator. Sci Signal 9: ra1.

60. Fisher LM, Pan XS (2008) Methods to assay inhibitors of DNA gyrase and topoisomerase IV activities. Methods Mol Med 142: 11-23.

61. Mdluli K, Ma Z (2007) Mycobacterium tuberculosis DNA gyrase as a target for drug discovery. Infect Disord Drug Targets 7: 159-168.

62. Lujan SA, Guogas LM, Ragonese H, Matson SW, Redinbo MR (2007) Disrupting antibiotic resistance propagation by inhibiting the conjugative DNA relaxase. Proc Natl Acad Sci U S A 104: 12282-12287.

63. Molnár J, Mándi Y, Spengler G, Haszon I, Turi S, et al (2014) Synergism between antiplasmid promethazine and antibiotics In Vitro and In Vivo. Biochemistry \& Pharmacology 3: 4.

64. Schelz Z, Molnar J, Hohmann J (2006) Antimicrobial and antiplasmid activities of essential oils. Fitoterapia 77: 279-285.

65. Gurunathan S, Han JW, Kwon DN, Kim JH (2014) Enhanced antibacterial and anti-biofilm activities of silver nanoparticles against Gram-negative and Grampositive bacteria. Nanoscale Res Lett 9: 373

66. Schulze CJ, Bray WM, Woerhmann MH, Stuart J, Lokey RS, Linington RG (2013) "Function-first" lead discovery: mode of action profiling of natura product libraries using image-based screening. Chem Biol 20: 285-295.

67. Lewis K (2013) Platforms for antibiotic discovery. Nat Rev Drug Discov 12 371-387.

68. Bueno J (2015) chapter 2 - Antimicrobial models in nanotechnology: from the selection to application in the control and treatment of infectious diseases: Nanotechnology in Diagnosis, Treatment and Prophylaxis of Infectious Diseases. Academic Press, Boston.

69. Escaich S (2010) Novel agents to inhibit microbial virulence and pathogenicity. Expert Opin Ther Pat 20: 1401-1418.

70. Fernebro J (2011) Fighting bacterial infections-future treatment options. Drug Resist Updat 14: 125-139.

71. Nathan C (2012) Fresh approaches to anti-infective therapies. Sci Transl Med 4: $140 \mathrm{sr} 2$.

72. Wong WR, Oliver AG, Linington RG (2012) Development of antibiotic activity profile screening for the classification and discovery of natural product antibiotics. Chem Biol 19: 1483-1495.

73. Blondeau JM (2009) New concepts in antimicrobial susceptibility testing: the mutant prevention concentration and mutant selection window approach. Vet Dermatol 20: 383-396.

74. Zhang Y (2014) Persisters, persistent infections and the Yin-Yang model. Emerg Microbes Infect 3: e3.

75. Maisonneuve E, Gerdes K (2014) Molecular mechanisms underlying bacteria persisters. Cell 157: 539-548.

76. Helaine S, Kugelberg E (2014) Bacterial persisters: formation, eradication, and experimental systems. Trends Microbiol 22: 417-424.

77. Wood TK, Knabel SJ, Kwan BW (2013) Bacterial persister cell formation and dormancy. Appl Environ Microbiol 79: 7116-7121.

78. Goneau LW, Yeoh NS, MacDonald KW, Cadieux PA, Burton JP, et al (2014) Selective target inactivation rather than global metabolic dormancy causes antibiotic tolerance in uropathogens. Antimicrob Agents Chemother 58: 20892097

79. Bueno J (2012) Antitubercular In Vitro Drug Discovery: Tools for Begin the Search: Understanding Tuberculosis - New Approaches to Fighting Against Drug Resistance. InTech, Rijeka.

80. Desalermos A, Muhammed M, Glavis-Bloom J, Mylonakis E (2011) Using C. elegans for antimicrobial drug discovery. Expert Opin Drug Discov 6: 645-652.

81. Moy TI, Ball AR, Anklesaria Z, Casadei G, Lewis K, et al. (2006) Identification of novel antimicrobials using a live-animal infection model. Proc Natl Acad Sci U S A 103: 10414-10419. 
Citation: Bueno J (2016) Antimicrobial Adjuvants Drug Discovery, the Challenge of Avoid the Resistance and Recover the Susceptibility of MultidrugResistant Strains. J Microb Biochem Technol 8: 169-176. doi: 10.4172/1948-5948.1000281

82. Moy TI, Conery AL, Larkins-Ford J, Wu G, Mazitschek R, et al. (2009) Highthroughput screen for novel antimicrobials using a whole animal infection model. ACS Chem Biol 4: 527-533.

83. Conery AL, Larkins-Ford J, Ausubel FM, Kirienko NV (2014) High-throughput screening for novel anti-infectives using a $\mathrm{C}$. elegans pathogenesis model. Curr Protoc Chem Biol 6: 25-37.

84. Viveiros M, Amaral L (2001) Enhancement of antibiotic activity against polydrug resistant Mycobacterium tuberculosis by phenothiazines. Int J Antimicrob Agents 17: 225-228.

85. Dastidar S, Kristiansen J, Molnar J, Amaral L (2013) Role of phenothiazines and structurally similar compounds of plant origin in the fight against infections by drug resistant bacteria. Antibiotics 2: 58-72.

86. Kristiansen JE, Thomsen VF, Martins A, Viveiros M, Amaral L (2010) Nonantibiotics reverse resistance of bacteria to antibiotics. In Vivo 24: 751-754.

87. Martins M, Dastidar SG, Fanning S, Kristiansen JE, Molnar J, et al (2008) Potential role of non-antibiotics (helper compounds) in the treatment of multidrug-resistant Gram-negative infections:mechanisms for their direct and indirect activities. Int J Antimicrob Agents 31:198-208.

88. Coutinho HD, Costa JG, Lima EO, Falcão-Silva VS, Siqueira-Júnior JP (2008) Enhancement of the antibiotic activity against a multiresistant Escherichia coli by Mentha arvensis $L$. and chlorpromazine. Chemotherapy. 54: 328-330

89. Yap PS, Yiap BC, Ping HC, Lim SH (2014) Essential oils, a new horizon in combating bacterial antibiotic resistance. Open Microbiol J 8: 6-14.

90. de Oliveira AD, Galvao Rodrigue FF, Douglas Melo Coutinho H, da Costa JG, de Menezes IR (2014) Chemical composition, modulatory bacterial resistance and antimicrobial activity of essential oil the Hyptis martiusii benth by direct and gaseous contact. Jundishapur J Nat Pharm Prod 9: e13521.

91. So AD, Gupta N, Brahmachari SK, Chopra I, Munos B, et al. (2011) Towards new business models for R\&D for novel antibiotics. Drug Resist Updat 14: 88-94.

92. Silver LL (2011) Challenges of antibacterial discovery. Clin Microbiol Rev 24: 71-109.

93. Butler D (2008) Translational research: crossing the valley of death. Nature 453: $840-842$.

94. Pan SY, Pan S, Yu ZL, Ma DL, Chen SB, et al. (2010) New perspectives on innovative drug discovery: an overview. J Pharm Pharm Sci 13: 450-471.

95. Pienta KJ (2010) Successfully accelerating translational research at an academic medical center: the University of Michigan-Coulter translational research partnership program. Clin TransI Sci 3: 316-318.
96. Macarron R, Banks MN, Bojanic D, Burns DJ, Cirovic DA, et al. (2011) Impact of high-throughput screening in biomedical research. Nat Rev Drug Discov 10: 188-195.

97. Kingston DG (2011) Modern natural products drug discovery and its relevance to biodiversity conservation. J Nat Prod 74: 496-511.

98. Singh SB, Pelaez F (2008) Biodiversity, chemical diversity and drug discovery Prog Drug Res 65: 141, 143-174.

99. Zakeri B, Lu TK (2013) Synthetic biology of antimicrobial discovery. ACS Synth Biol 2: 358-372.

100.de Castro AP, Fernandes GDR, Franco OL (2014) Insights into nove antimicrobial compounds and antibiotic resistance genes from soil metagenomes. Front Microbiol 5: 489.

101. Liu X, Ashforth E, Ren B, Song F, Dai H, et al. (2010) Bioprospecting microbial natural product libraries from the marine environment for drug discovery. $J$ Antibiot (Tokyo) 63: 415-422.

102. Rahman MA, Islam MZ, Islam MA (2011) Antibacterial activities of actinomycete isolates collected from soils of rajshahi, bangladesh. Biotechnol Res Int 2011: 857925.

103. Berdy J (2012) Thoughts and facts about antibiotics: where we are now and where we are heading. J Antibiot (Tokyo) 65: 385-395.

104. Joseph B, Priya RM (2011) Bioactive compounds from endophytes and their potential in pharmaceutical effect: a review. Am J Biochem Mol Biol 1: 291-309.

105. Warnke PH, Becker ST, Podschun R, Sivananthan S, Springer IN, et al (2009) The battle against multi-resistant strains: Renaissance of antimicrobial essential oils as a promising force to fight hospital-acquired infections. J Craniomaxillofac Surg 37: 392-397.

106. Bueno J (2012) In vitro antimicrobial activity of natural products using minimum inhibitory concentrations, looking for new chemical entities or predicting clinical response. Med Aromat Plants 1: 113.

107. Peleg AY, Jara S, Monga D, Eliopoulos GM, Moellering RC Jr, et al. (2009) Galleria mellonella as a model system to study Acinetobacter baumannii pathogenesis and therapeutics. Antimicrob Agents Chemother 53: 2605-2609.

108. Baquero F, Coque TM, de la Cruz F (2011) Ecology and evolution as targets: the need for novel eco-evo drugs and strategies to fight antibiotic resistance. Antimicrob Agents Chemother 55: 3649-3660.

109. Sengupta S, Chattopadhyay MK, Grossart HP (2013) The multifaceted roles of antibiotics and antibiotic resistance in nature. Front Microbiol 4: 47.

110. Bollenbach T (2015) Antimicrobial interactions: mechanisms and implications for drug discovery and resistance evolution. Curr Opin Microbiol 27: 1-9. 